\title{
A Comparative STUdy ON IMAgE COMPRESSION USING HALFTONING BASED BLOCK TRUNCATION CODING FOR COLOR IMAGE
}

\author{
Meharban M.S ${ }^{1}$ and Priya $S^{2}$ \\ ${ }^{1}$ M.Tech Student, Dept. of Computer Science, Model Engineering College \\ ${ }^{2}$ Associate Professor, Dept. of Computer Science, Model Engineering College
}

\begin{abstract}
In this paper scrutinizes image compression using Halftoning Based Block Truncation Coding for color image. Many algorithms were selected likely the original Block Truncation coding, Ordered Dither Block Truncation Coding, Error Diffusion Block Truncation Coding, and Dot Diffused Block Truncation Coding. These above techniques are divided image into non overlapping blocks. BTC acts as the basic compression technique but it exhibits two disadvantages such as the false contour and blocking effect. Hence halftoning based block truncation coding (HBTC) is used to overcome the two issues. Objective measures are used to evaluate the image degree of excellence such as Peak Signal to Noise Ratio, Mean Square Error, Structural Similarity Index and Compression Ratio. At the end, conclusions have shown that the Dot Diffused Block Truncation Coding algorithm outperforms the Block Truncation Coding as well as Error Diffusion Block Truncation Coding.
\end{abstract}

\section{KEYWORDS}

Halftoning, Image Compression, Block Truncation Coding (BTC), Error Diffusion, Dot Diffusion

\section{INTRODUCTION}

In recent years, the development of multimedia product is rapidly growing which contributes to insufficient bandwidth of network and storage. Thus the theory of image compression gains more importance for reducing the storage space and transmission bandwidth needed. Digital halftoning is a technique for converting continuous-tone images into two-tone image[8]. The results can resemble the original images when viewed from a distance by involving the low-pass nature of the Human Visual System (HVS). Today, digital halftoning plays a key role in almost every discipline that involves printing and displaying. All newspapers, magazines, and books are printed with digital halftoning [15].For color image separate halftone is generated for cyan, magenta, yellow and black .Each halftone screen is rotated to form a pat-tern called rosette. Effective digital halftoning can substantially improve the quality of rendered images at minimal cost [8].The major issues in choosing a halftoning technique are image quality and amount of computation. Some of the major Halftoning method that has been developed so far includes the ordered dithering, Error diffusion and Dot diffusion.

Block Truncation Coding (BTC) is a lossy image compression technique which uses moment 
preserving quantization method for compressing digital gray scale images as well as color image. BTC has been used for many years for compressing digital monochrome images. BTC has been used for many years for compressing digital monochrome images. It is a simple and lossy image compression technique.

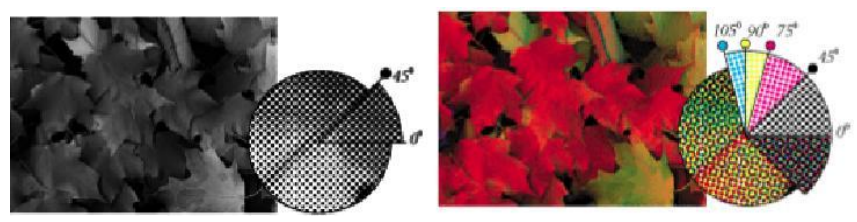

Fig .1 Enlarged details of halftone dot pattern for

(a) Grayscale image (b) Color image

The BTC method preserves the block mean and standard deviation [1].The simplest way to extend BTC to color image is to apply BTC to each color plane independently[10][2].The disadvantage of this method is that three bit plane are needed hence the compression ratios achievable are low.

\section{BLOCK TRUNCATION CODING}

Block Truncation Coding (BTC) is a lossy image compression technique which uses moment preserving quantization method for compressing digital gray scale images as well as color image [2]. In block truncation coding (BTC), the original image is divided into fixed-size non overlapping blocks of size M N [1] .The block size chosen is usually small to avoid the edge blurring and blocking effect. Each block is independently coded using a two level (1-bit) quantizer. The two values preserve the first and the second moment characteristic of the original block. BTC does not provide a higher gain than any of the modern image compressing algorithms like JPEG or JPEG-2000, but it is much lesser complex [5] .While BTC based image compression method provide low computational complexity ,the method also has the issue of degradation of the image quality when compared to other compression technique. However BTC based image compression also suffer from two major issues namely blocking effect and false contours [6].

\section{HALfTONing Methods}

Three common methods for generating digital halftoning images are

1. Dithering:Common technique used for generating digital halftoning images is dithering. Dithering creates an output image with the same number of dots as the number of pixels in the source image. Dithering can be thought of as thresholding the source image with a dither matrix. The matrix is laid repeatedly over the source image. Wherever the pixel value of the image is greater than the value in the matrix, a dot on the output image is filled.

2. Error diffusion:Error diffusion is another technique used for generating digital half toned images. It is often called spatial dithering. Error diffusion sequentially traverses each pixel of the source image. Each pixel is compared to a threshold. If the pixel value is 
higher than the threshold, a 255 is outputted; otherwise, a 0 is outputted. The error, the difference between the input pixelvalue and the output value is dispersed to nearby neighbors.

3. Dot diffusion:Dot diffusion method for halftoning, is an attractive method which attempts to retain the good features of error diffusion while offering substantial parallelism. The dot diffusion method for halftoning has only one design parameter called the class matrix.

\section{Halftoning BaSed Block Truncation Coding}

Halftoning based block truncation coding is an extended compression technique derived from BTC scheme in which the BTC bitmap image is replaced with halftone image. The main difference between BTC and HBTC is on the image block quantizer determination .In contrast to the BTC scheme which tries to maintain its mean value and standard deviation in image block. The HBTCquantizer is simply obtained from the minimum and maximum value found in an image block. Error diffusion based BTC offers an improved image quality. Ordered dithering based BTC is used when the main requirement is performance efficiency .Dot diffusion based method provide a balance of the above two requirements, better visual quality as well as a better performance efficiency [5][9][10].The three methods described above all provide a proper solution to the problems in a traditional BTC, such us blocking effect and false contours.

\subsection{ORdered Dither BLOCK TrunCation CODing}

The dithering-based BTC, namely Ordered Dither Block Truncation Coding (ODBTC) is an example of HBTC. In which bit pattern configuration of the bitmap is generated from the dithering approach (void-and-cluster Halftoning) .In encoding stage, the ODBTC scheme utilizes the dither array Look-Up-Table (LUT) to speed up the processing speed. The dither array in ODBTC method substitutes the fixed average value as the threshold value for the generation of bitmap image [6]. The extreme values in ODBTC are simply obtained from the minimum and maximum value found in the image blocks. ODBTC offers high efficiency and low computational complexity. The quantization error cannot be compensated with the ordered dithering halftoning and thus the ODBTC yields lower image quality compared to that of the EDBTC.

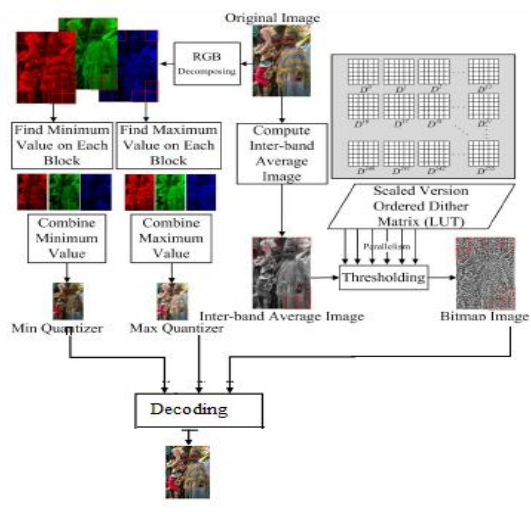

Fig 2. Block or ODBTC 


\subsection{ERror Diffusion BLOCK TRUnCATION CODING}

Error diffusion is a method that provides better visual quality of image .Error diffusion based BTC does this while also compressing the image .In this method, the inherent dithering property of error diffusion is to deal with the problem of false contour. Similar to the BTC scheme, EDBTC looks for a new representation (two quantizer and bitmap image) for reducing the storage requirement. The EDBTC bitmap image is constructed by considering the quantized error which diffuses to the nearby pixels to compensate the overall brightness .EDBTC employs the error kernel to generate the representative bitmap image. Fig 4,5 shows the error diffusion kernels for Floyd-Steinberg, Stucki, Jarvis, and Stevenson .Different error kernel yield different halftoning pattern. Error diffusion strategy effectively removes the annoying blocking effect and false contour, while maintaining the low computational complexity. The prolonged processing time is still an issue [5][10].

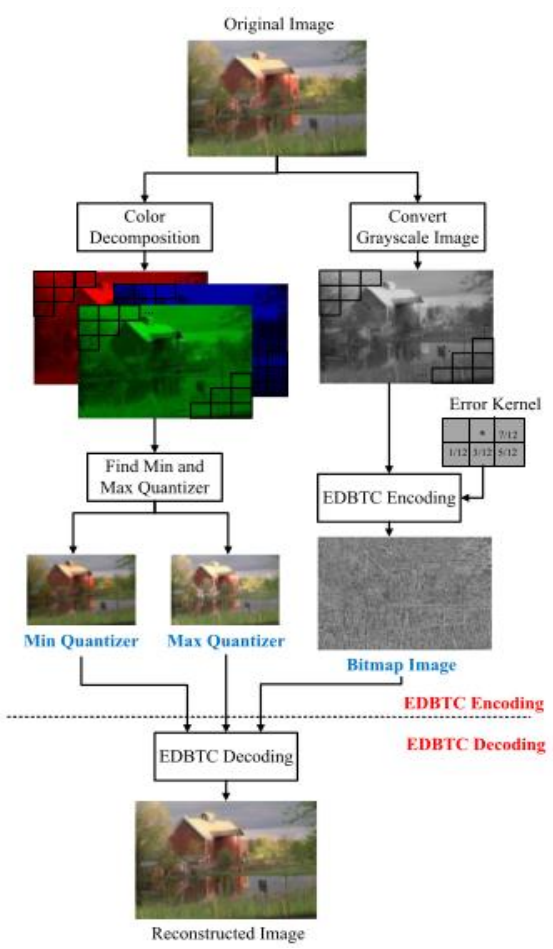

Fig. 4 Error Kernels (a) Floyd-Steinberg (b) Stuck

\begin{tabular}{|l|l|l|l|l|}
\hline & & $*$ & $7 / 48$ & $5 / 48$ \\
\hline $3 / 48$ & $5 / 48$ & $7 / 48$ & $5 / 48$ & $3 / 48$ \\
\hline $1 / 48$ & $3 / 48$ & $5 / 48$ & $3 / 48$ & $1 / 48$ \\
\hline
\end{tabular}

\begin{tabular}{|c|c|c|c|c|}
\hline & & $*$ & $5 / 32$ & $3 / 32$ \\
\hline $2 / 32$ & $4 / 32$ & $5 / 32$ & $4 / 32$ & $2 / 32$ \\
\hline $0 / 32$ & $2 / 32$ & $3 / 32$ & $2 / 32$ & $0 / 32$ \\
\hline
\end{tabular}

\begin{tabular}{|l|l|l|}
\hline & $*$ & $7 / 16$ \\
\hline $3 / 16$ & $5 / 16$ & $1 / 16$ \\
\hline
\end{tabular}

\begin{tabular}{|l|c|c|c|l|}
\hline & & & $8 / 42$ & $4 / 42$ \\
\hline $2 / 42$ & $4 / 42$ & $8 / 42$ & $4 / 42$ & $2 / 42$ \\
\hline $1 / 42$ & $2 / 42$ & $4 / 42$ & $2 / 42$ & $1 / 42$ \\
\hline
\end{tabular}

Fig. 5 Error Kernels (c) Jarvis (d) Sierr 

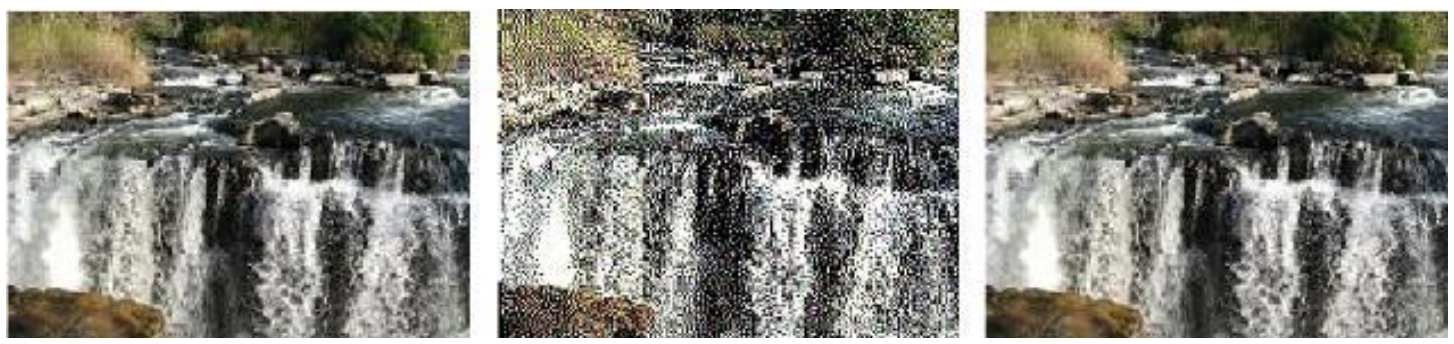

Fig. 5.Image quality comparison on different EDBTC kernel

(a) Original (b) Floyd (c) Stucki

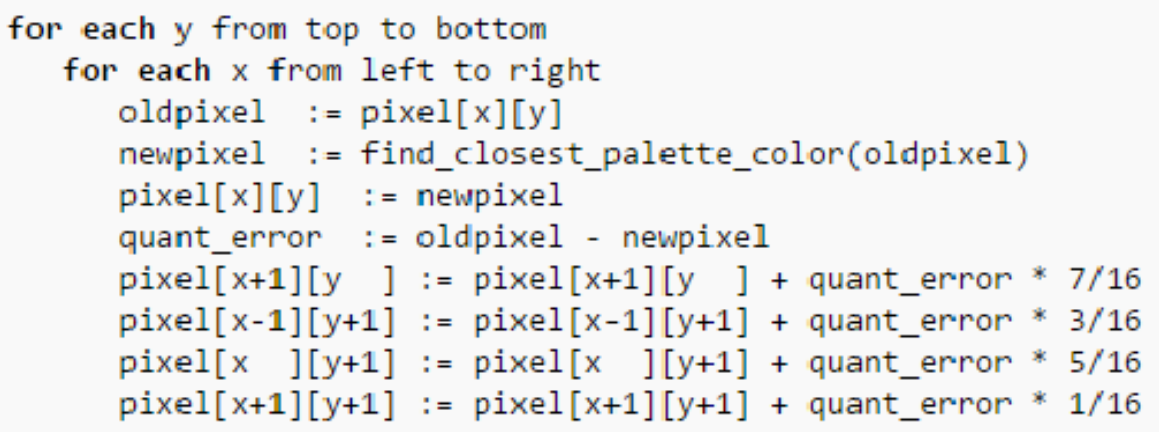

Fig. 6 Algorithm for error diffusion BTC

\subsection{Dot DifFused BLOCK TRUNCATION CODING}

Dot diffusion based BTC provides better visual quality and performance efficiency compared to Error diffusion based BTC [4].Here the natural parallelism of dot diffusion is utilized to obtain better processing efficiency .Better image quality is achieved by co-optimizing the diffused matrix and class matrix of the dot diffusion. It also provides better image quality compared to Ordered Dithering based BTC as well. The DDBTC effectively compresses an image by decomposing an image into two quantizers and a bitmap image. The DDBTC diffuses the quantization error of the current processed pixel into its neighboring pixels using the diffused matrix and class matrix concurrently to generate the bitmap image .High dynamic range can easily destroy the blocking effect and false contour.

Class Matrix of size $4 * 4$

\begin{tabular}{|l|l|l|l|}
\hline .2716 & \multicolumn{1}{l|}{1} & .2716 \\
\hline 1 & & $\mathrm{X}$ & \multicolumn{1}{l|}{1} \\
\hline \multicolumn{2}{|l|}{2716} & 1 & \multicolumn{2}{l|}{} \\
\hline 8 & 11 & 6 & 1 \\
\hline 14 & 2 & 4 & 12 \\
\hline 5 & 9 & 7 & 3 \\
\hline 13 & 5 & 0 & 10 \\
\hline
\end{tabular}




\section{EXPERIMENTAL RESULT}

\subsection{OBJECTIVE QUALiTy MEASURES COMPARISON}

Objective measures are used to evaluate the image degree of excellence such as Peak Signal to Noise Ratio, Mean Square Error, Compression Ratio and Structural Similarity Index (SSIM).

The PSNR is the ratio between a signal's maximum power and the power of the signal's noise. By using the PSNR values the quality of reconstructed images is best described. Mathematically, PSNR is defined as:

$\mathrm{PSNR}=10 * \log 10\left[\frac{255^{2}}{\frac{1}{M * N} \sum \sum\left(f(m, n)-g(m, n)^{2}\right.}\right]$

Mean square error is a very useful measures as it gives an average value of the energy lost in the lossy compression of the original image .A very small MSE means the image is very closer to the original, $f(m, n)$ is the original image,$g(m, n)$ is the reconstructed image MSE is defined as.

$\mathrm{MSE}=\frac{1}{M * N} \sum \sum\left(f(m, n)-g(m, n)^{2}\right)$

The compression ratio is used to measure the ability of data compression by comparing the size of the image being compressed to the size of the original image.

Rate is another metric used to evaluate the performance of the compression algorithm. Rate gives the number of bits per pixel used to encode an image rather than abstract percentage.

Another category of image quality measures is based on the assumption that the human visual system is highly adapted to extract structural information from the viewing field [10]. The error sensitivity approach estimates perceived errors to quantify image degradations, while this approach considers image degradations as perceived structural information variation. The structural similarity index (SSIM) can be calculated as a function of three components: luminance, contrast and structure. 
$\operatorname{SSIM}(\mathrm{x} ; \mathrm{y})=[\mathrm{l}(\mathrm{x} ; \mathrm{y})]^{\alpha}[\mathrm{c}(\mathrm{x} ; \mathrm{y})]^{\beta}[\mathrm{s}(\mathrm{x} ; \mathrm{y})]^{\gamma}$

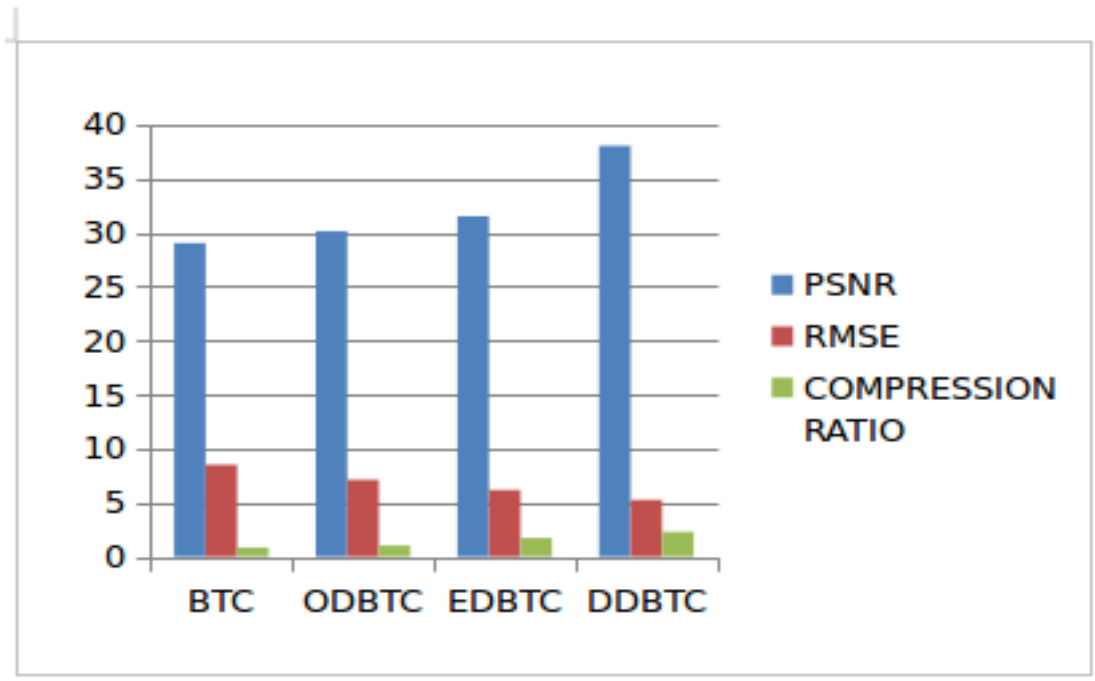

Fig .7 Objective image quality comparisons

Table I. Objective Image Quality Comparison

\begin{tabular}{|c||c|l|c|c|}
\hline Type of BTC & RMSE & PSNR & Compression Ratio & SSIM \\
\hline BTC & 7.52 & 29.03 & 0.8607 & 0.8734 \\
\hline ODBTC & 7.13 & 30.13 & 1.03611 & 0.93245 \\
\hline EDBTC & 6.17 & 31.52 & 1.73611 & 0.94076 \\
\hline DDBTC & 5.27 & 38.03 & 2.3000 & 0.95518 \\
\hline
\end{tabular}

The table illustrates the PSNR, MSE, SSIM and Compression Ratio values obtained for different halftoning based BTC. From the table as well as through visual inspection of the result images, it can be seen that Dot Diffused BTC provide better visual quality compared to other halftoning based BTC. 


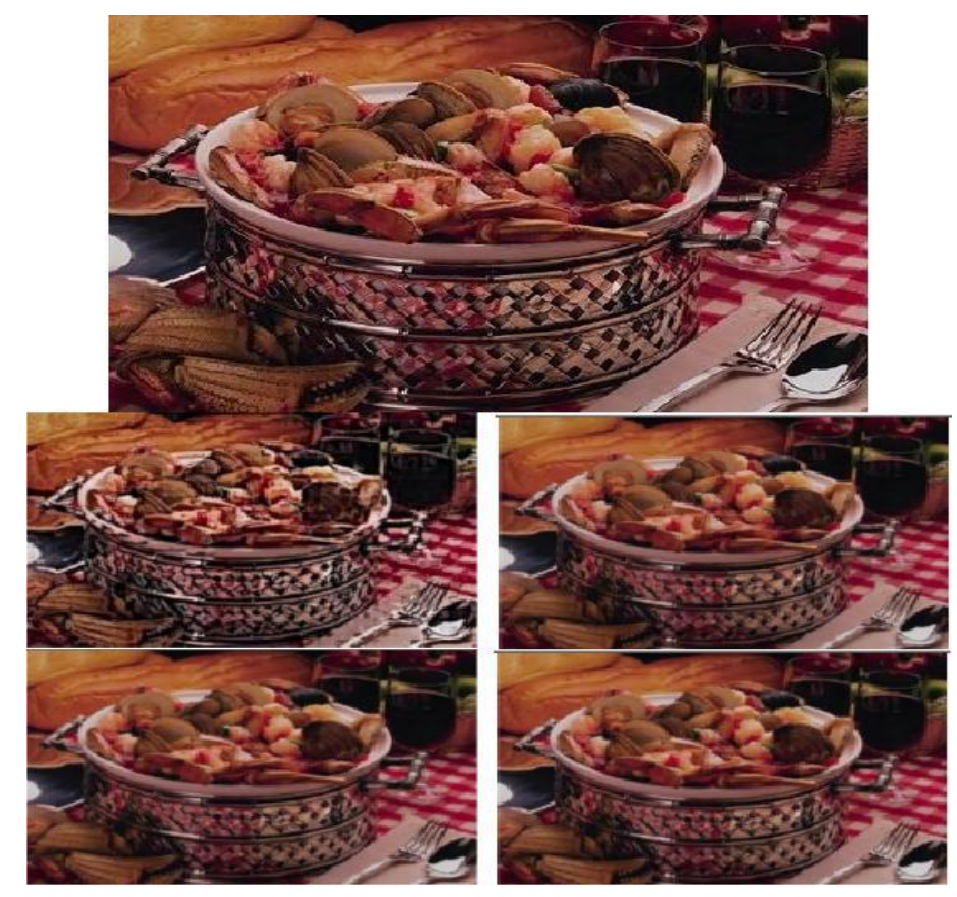

Fig.8.Image quality comparison on different HBTC (a)original,(b)BTC,(c)ODBTC,(d)EDBTC,(e)DDBTC

\section{Conclusion}

In this literature survey, image compression using Halftoning based Block Truncation coding for color image has been scrutinized, which can provide an excellent image quality and artifact free result such us inherent blocking effect and false contour artifact of the traditional BTC simultaneously. Four algorithms were selected specifically, the original block truncation coding (BTC), Ordered dither Block truncation coding (ODBTC), Error diffused block truncation coding (EDBTC) and Dot diffused Block truncation coding (DDBTC). Objectives measures are used to evaluate the image degree of excellence such as PSNR, MSE, SSIM and Compression Ratio, In this survey find out that halftoning based BTC not only applied for gray scale image it can be extended for color image .For future study, other color spaces can be explored for the image compression such as YCbCr, Lab color space, HSI etc.

\section{REFERENCES}

[1] E.J Delp and O.R .Mitchell. Image coding using block truncation coding. IEEE Transactions on Image Processing, 27(1):1335-1342, Sept 1979.

[2] G. Qiu. Color Image Indexing Using BTC IEEE Transactions on Image Processing, 12(1) , Jan. 2003.

[3] J.M Guo High efficiency ordered dither block truncation with dither array LUT and its scalable coding application IEEE Transactions on Image Processing, 20(1):97-110, Jan 2010.

[4] J.M Guo and Y.F.Liu. Improved Block Truncation Coding using Optimized Dot Diffusion. IEEE Transactions on Image Processing, 2(1):1269-1275, Jan 2014. 
[5] J.M Guo and Y.F.Liu. Improved Block Truncation Coding using modified error diffusion. IEEE Transactions on Image Processing, 44(7):462-464, Mar 2008.

[6] J.M Guo and Y.F.Liu. Improved Block Truncation Coding Based on the Void-and-Cluter Dithering Approach. IEEE Transactions on Image Processing, 18(1):211-213, Jan 2009.

[7] David Saloman. Data Compression the Complete Reference. Fourth Edition.

[8 ]R Ulichney. Digital Halftoning. Cambridge,USA:: MIT Press, 1987

[9] M.Kamel,C.T.Sun and G.Lian. Image compression by variable block truncation coding with optimal threshold. IEEE Transactions on Signal Processing, 39(1):208-212, Jan 1991.

[10] Jing-Ming Guo and Yun-Fu Liu. High Capacity Data Hiding for Error-Diffused Block Truncation Coding.. IEEE Transactions on Signal Processing, 21(12):4808-4818, December 2012.

[11] S.Vimala, P.Uma, B. Abidha. Improved Adaptive Block Truncation Coding for Image Compression International Journal of Computer Applications, 19(7):975-988, April 2011 .

[12] M. D. Lema and O. R. Mitchell. High Absolute moments block truncation coding and its application to color images, IEEE Trans. Commun, 4(32):1148-1157, Oct. 1984

[13] V. R. Udpikar and J. P. Raina. High BTC image coding using vector quantization, IEEE Trans. Commun, 4(35):352-358, Sept. 1987.

[14] H. R. Kang Digital Color Halftoning New York: 1999.

[15] S.Vimala, P.Uma, B. Abidha. Improved Adaptive Block Truncation Coding for Image Compression International Journal of Computer Applications, 19(7):975-988, April 2011

[16] V. R. Udpikar and J. P. Raina. High BTC image coding using vector quantization, IEEE Trans. Commun, 4(35):352-358, Sept. 1987.

[17] P. Franti, and T. Kaukoranta, Binary vector quantizer design using soft centroids, Signal Proc Image Comm, vol. 14, no. 9, pp. 677-681, 1999.

[18] M.Kamel,C.T.Sun and G.Lian. Image compression by variable block truncation coding with optimal threshold. IEEE Transactions on Signal Processing, 39(1):208-212, Jan 1991.

[19] Jing-Ming Guo and Yun-Fu Liu. High Capacity Data Hiding for Error-Diffused Block Truncation Coding. IEEE Transactions on Signal Processing, 21(12):4808-4818, December 2012.

[20] S.Vimala, P.Uma, B. Abidha. Improved Adaptive Block Truncation Coding for Image Compression. International Journal of Computer Applications, 19(7):975-988, April 2011

[21] C. S. Huang and Y. Lin. Hybrid block truncation coding, IEEE Trans. Signal Process, 4(12):328-330, Dec 1997

[22] M. D. Lema and O. R. Mitchell. High Absolute moment blocks truncation coding and its application to color images, IEEE Trans. Commun, 4(32):1148-1157, Oct. 1984.

[23] H. R. Kang. Digital Color Halftoning, New York: 1999.

[24] Smen Forchhammer and Kim S. Jensen. Data Compression of Scanned Halftone Images, IEEE Trans. Commun, 4(42):213-238, Mar. 1997.

[25] Arup Kumar Pal. An efficient codebook initialization approach for LBG algorithm, International Journal of Computer Science, Engineering and Applications (IJCSEA), Vol.1, No.4, August 2011. 
International Journal on Cybernetics \& Informatics (IJCI) Vol. 5, No. 2, April 2016

\section{AUTHORS}

Meharban M S was born in Perumbavoor in 1993. She received BE in Information technology from Cochin University Of science and technology in 2014. She is currently an M.Tech Candidate in computer science from model engineering college, Thrikkakara in 2016. She is working as part time faculty at IGNOU.

Dr. Priya S Obtained her BTech in Computer Science \& Engineering from Kerala University, MTech in Computer Science \& Engineering from Pondicherry University, Pondicherry India and $\mathrm{PhD}$ in Information \& Communication Engineering from Anna University, Chennai, India. She is currently working as Associate Professor in the Department of Computer Science \& Engineering in Govt Model Engineering College, Thrikkakara, Ernakulam, and Kerala, India. Her experience as a faculty is more than 18 years as of now. She is a life member of IST. 